\title{
CAPLIB: A New Program Library for the Modeling and Analysis of Icosahedrally Symmetric Viral Capsids
}

Shigetaka Yoneda,*il Yukina Hara-Yamada, Aya Kosugi, Maiko Nanao, Takami Saito, Shunsuke Sato, Nozomu Yamada, and Go Watanabe

School of Science, Kitasato University, 1-15-1 Kitasato, Minami-Ku, Sagamihara-Shi, Kanagawa 252-0373, Japan

\section{Supporting Information}

ABSTRACT: A new program library named "CAPLIB" was developed for the modeling and analysis of icosahedrally symmetric virus capsids. CAPLIB is equipped with the mathematical data of 60 rotation matrices of icosahedral symmetry, 15 planes bisecting the entire capsid structure, and a table summarizing how the 60 asymmetric units (cells) are partitioned by the planes. CAPLIB contains the function to determine the cell numbers of atoms from the atomic positions and the function to determine the rotation axes and angles from the rotation matrices. Using CAPLIB, it is possible to generate the structure of any selected protein unit within the entire capsid by rotating a single protein unit structure. CAPLIB can classify Protein Data Bank files of capsids with the directions of rotation axes, rotate the protein structure onto the standard position, and perform various deformations of the entire capsid. The interface to the molecular graphics software, PyMOL, was also developed for efficient modeling of capsids.

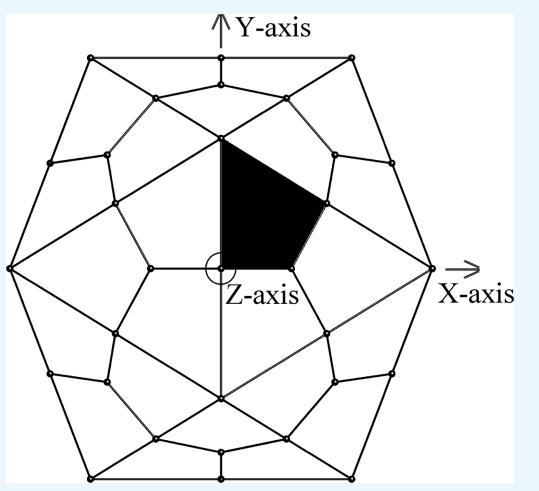

\section{INTRODUCTION}

Viral capsids are symmetric assemblies of identical protein units in icosahedral symmetry or helical symmetry. Especially, icosahedral symmetry of capsids has intrigued many mathematical and computational studies because against the straightforward prediction, an icosahedrally symmetric capsid is not necessarily composed of 60 identical proteins: several
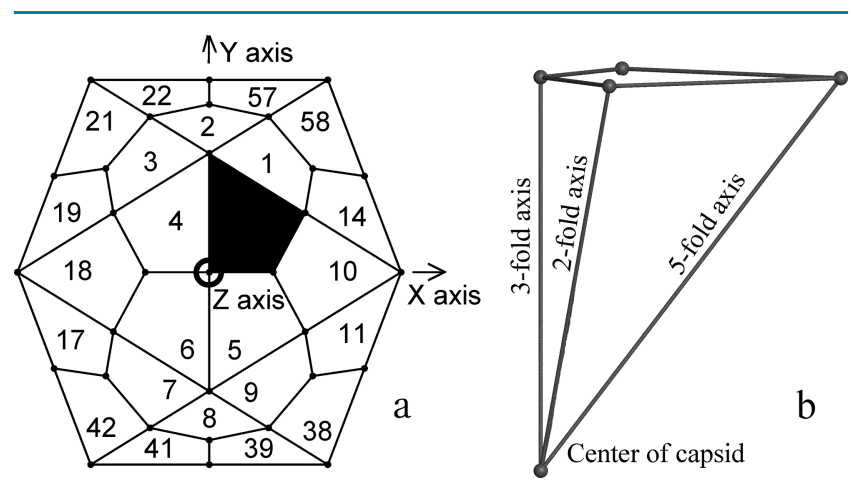

Figure 1. Asymmetric unit (cell) for icosahedral symmetry. (a) A regular icosahedron surface is seen from a 2 -fold rotation axis ( $Z$-axis). The surface of the icosahedron is composed of 20 regular triangles, each of which is divided into the three identical oblique quadrangles. The black oblique quadrangle denotes the cell labeled 0 . The integers denote the numbers of other cells. (b) A cell is surrounded by the four partition planes. The edges of the cell are a 5-fold axis, 3 -fold axis, and two 2 -fold axes. The apex of the cell is the center of the capsid. The volume of a cell is infinitely large, but we sketched only the bottom of the pyramid for clarity. In particular, a cross section of the cell with the icosahedron surface is shown.
Table 1. Rotation Matrices A, B, C, and D

$$
\begin{array}{ll}
\text { matrix } & \left.\begin{array}{lll}
-(1+\sqrt{5}) / 4 & -1 / 2 & (-1+\sqrt{5}) / 4 \\
-1 / 2 & (-1+\sqrt{5}) / 4 & -(1+\sqrt{5}) / 4 \\
(-1+\sqrt{5}) / 4 & -(1+\sqrt{5}) / 4 & -1 / 2
\end{array}\right) \\
\mathrm{B} & \left(\begin{array}{lll}
-1 / 2 & (1-\sqrt{5}) / 4 & -(1+\sqrt{5}) / 4 \\
(1-\sqrt{5}) / 4 & -(1+\sqrt{5}) / 4 & 1 / 2 \\
-(1+\sqrt{5}) / 4 & 1 / 2 & (-1+\sqrt{5}) / 4
\end{array}\right) \\
\mathrm{C} & \left(\begin{array}{lll}
(1-\sqrt{5}) / 4 & -(1+\sqrt{5}) / 4 & 1 / 2 \\
(1+\sqrt{5}) / 4 & -1 / 2 & (1-\sqrt{5}) / 4 \\
1 / 2 & (-1+\sqrt{5}) / 4 & (1+\sqrt{5}) / 4
\end{array}\right) \\
\mathrm{D} & \left(\begin{array}{lll}
(-1+\sqrt{5}) / 4 & -(1+\sqrt{5}) / 4 & 1 / 2 \\
(1+\sqrt{5}) / 4 & 1 / 2 & (-1+\sqrt{5}) / 4 \\
-1 / 2 & (-1+\sqrt{5}) / 4 & (1+\sqrt{5}) / 4
\end{array}\right)
\end{array}
$$

identical proteins can make a protein unit; the number of the composing proteins of a capsid is generally 60 multiplied by $T$, where $T$ is the integer named "triangulation number". By considering embeddings of the surface of an icosahedron into a hexagonal lattice, Casper and Klug have proposed the principle of quasi equivalence to explain the mathematics of this

Received: February 15, 2018

Accepted: April 13, 2018

Published: April 24, 2018 


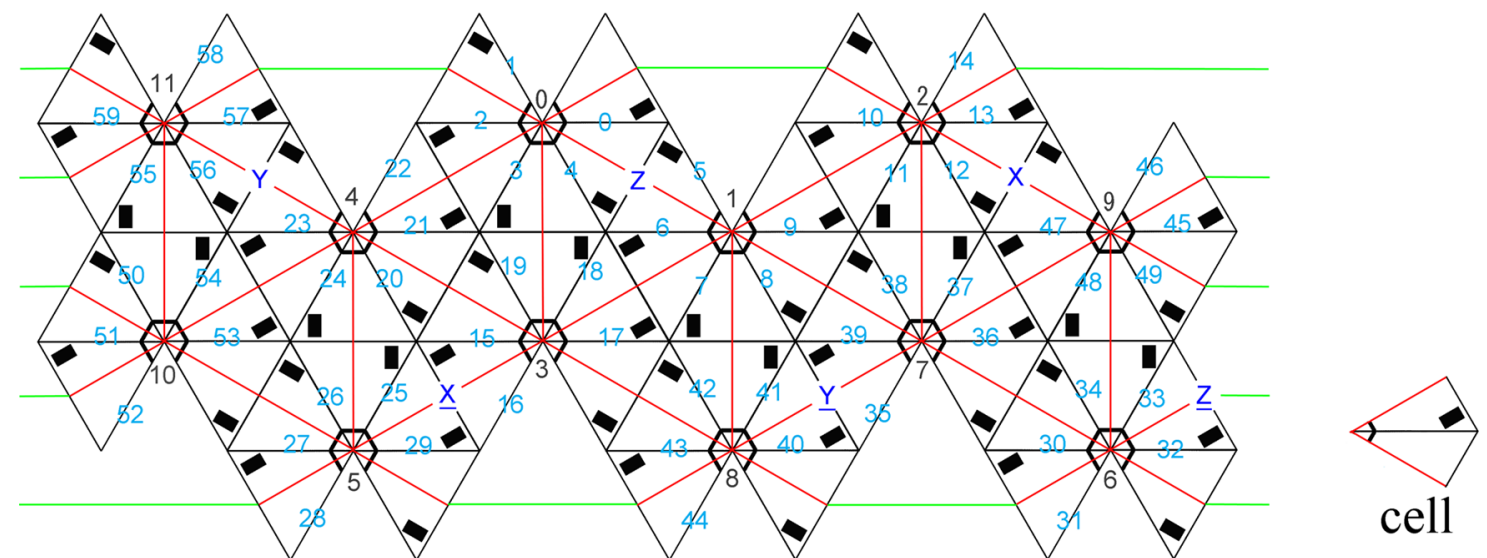

Figure 2. Development of the icosahedron surface. The 20 regular triangles are drawn with red lines. A three-dimensional icosahedron can be folded using the red lines for mountain creases. Each regular triangle is divided into three identical small quadrangles, of which the shape is shown in the inset. The quadrangle is an asymmetric unit of icosahedral symmetry and is used for the cell in this study. The intersection points of the red lines depict the 5-fold axes, those of the black lines depict the 3-fold axes, and those of the red lines and black lines depict the 2-fold axes. The small filled black rectangles are added only to indicate the orientations of the cells. The symbols $X, Y$, and $Z$ in blue denote the $X$-, $Y$-, and $Z$-axes, respectively, and the underlined $\underline{X}, \underline{Y}$, and $\underline{Z}$ denote their inverse directions. The cell numbers (from 0 to 59) are denoted in light blue, and the 5-fold axis numbers (from 0 to 11 ) are denoted in black. Each of the green lines connects the same position in the three-dimensional space.

\section{Table 2. Normal Vectors of Partition Planes}

$\begin{array}{cl}\text { plane } & \text { normal vector } \\ 0 & { }^{\mathrm{t}}(-(1+\sqrt{ } 5) / 4,0,0) \\ 1 & { }^{\mathrm{t}}(-(1+\sqrt{ } 5) / 8,1 / 4,(3+\sqrt{ } 5) / 8) \\ 2 & { }^{\mathrm{t}}(1 / 4,(3+\sqrt{ } 5) / 8,(1+\sqrt{ } 5) / 8) \\ 3 & { }^{\mathrm{t}}(1 / 4,(3+\sqrt{ } 5) / 8,-(1+\sqrt{ } 5) / 8) \\ 4 & { }^{\mathrm{t}}(-(1+\sqrt{ } 5) / 8,1 / 4,-(3+\sqrt{ } 5) / 8) \\ 5 & { }^{\mathrm{t}}((3+\sqrt{ } 5) / 8,(1+\sqrt{5}) / 8,-1 / 4) \\ 6 & \mathrm{t}(0,0,-(1+\sqrt{ } 5) / 4) \\ 7 & { }^{\mathrm{t}}(-(3+\sqrt{ } 5) / 8,-(1+\sqrt{ } 5) / 8,-1 / 4) \\ 8 & { }^{\mathrm{t}}(-(1+\sqrt{ } 5) / 8,-1 / 4,(3+\sqrt{ } 5) / 8) \\ 9 & { }^{\mathrm{t}}((1+\sqrt{ } 5) / 8,1 / 4,(3+\sqrt{ } 5) / 8) \\ 10 & { }^{\mathrm{t}}(-1 / 4,(3+\sqrt{ } 5) / 8,(1+\sqrt{ } 5) / 8) \\ 11 & { }^{\mathrm{t}}(-(3+\sqrt{ } 5) / 8,(1+\sqrt{ } 5) / 8,-1 / 4) \\ 12 & { }^{\mathrm{t}}(0,(1+\sqrt{ } 5) / 4,0) \\ 13 & { }^{\mathrm{t}}(-(3+\sqrt{ } 5) / 8,(1+\sqrt{ } 5) / 8,1 / 4) \\ 14 & \mathrm{t}(-1 / 4,(3+\sqrt{ } 5) / 8,-(1+\sqrt{ } 5) / 8)\end{array}$

$a^{a}$ Transposition of a vector is denoted by a superscript $\mathrm{t}$.

structural feature. Although their theory is widely used to classify virus species, the experimental structures of papovavirus capsids have been reported to be deviating from the theory and the more general viral tiling theory has been proposed. ${ }^{3}$ Many mathematical and theoretical studies including the tiling theory have thus been performed for the interests in the structure and energetics of icosahedrally symmetric viral capsids. ${ }^{4-7}$ In the field of crystallography, symmetry has been used to reduce the degree of freedom ${ }^{8}$ and many X-ray structures have been determined. Recently, some large viral capsids, including that of HIV-I, have been shown to be not perfectly symmetric ${ }^{9}$ but the structures of other small icosahedrally symmetric capsids are a mine of information for viral research, including the development of effective antiviral compounds. ${ }^{10}$ In the field of simulation, the rotational symmetry boundary condition (RSBC) has been proposed to accelerate molecular dynamics (MD) simulations of symmetric protein assemblies. ${ }^{11,12}$ The calculation of the energy of an entire capsid can be restricted by the RSBC to a single asymmetric unit (a computational cell or cell), and the calculation can be accelerated by 2 orders of magnitude. The RSBC has been implemented in the MD program APRICOT ${ }^{13}$ and used for studies of the icosahedrally symmetric capsids of rhinovirus, ${ }^{12}$ poliovirus, ${ }^{14}$ foot-and-mouse disease virus, ${ }^{15}$ and hepatitis B virus. ${ }^{16}$ An MD simulation has also been performed for a glycogen phosphorylase crystal in $\mathrm{P}_{3} \mathrm{2}_{1} 2$ symmetry using RSBC. ${ }^{17}$ Furthermore, the elaborate simulation in RSBC using a pentamer of the icosahedron for the computational cell has been performed and the effects of nonphysical microscopic symmetry imposed by RSBC near the axes have been deeply analyzed to estimate the validity and limitation of RSBC. ${ }^{18}$

On the above background, the authors present a new software library, CAPLIB, for the modeling and analysis on the structure of the icosahedrally symmetric capsid. Although this study is not directly related with simulation methods, the basis of RSBC is briefly described as follows because the concept of RSBC is used. Because a capsid is composed of 60 identical protein units, an atom is denoted by the combination of the atom number $k$ and the protein unit number $p$ shown as a subscript (i.e., $k_{p}$ ). Each atom is also specified by $k$ and the cell number $c$ shown as a superscript (i.e., $k^{c}$ ). $k$ ranges from 1 to $N$, where $N$ is the number of atoms in a protein unit. $N$ is also equal to the number of atoms within a cell. $p$ and $c$ take values from 0 to 59. Figure 1 shows the shape of the cell and the standard coordinate axes adopted in this study. The rotation matrix of icosahedral symmetry is written as $R[c]$. The position of an atom within cell 0 can be rotated with $R[c]$ onto the position of the then equivalent atom in cell $c$, as follows

$$
\mathbf{x}\left(k^{c}\right)=R[c] \mathbf{x}\left(k^{0}\right)
$$

where $\mathbf{x}$ denotes the atomic coordinates. Similarly, the position of an atom of protein unit 0 is rotated with $R[p]$ onto the position of the equivalent atom of protein unit $p$ as follows

$$
\mathbf{x}\left(k_{p}\right)=R[p] \mathbf{x}\left(k_{0}\right)
$$

The 60 rotation matrices, $R[p]$, make an algebraic group named the "icosahedral group", or, more precisely, "pure rotation 
ACS Omega

Article

Table 3. Complete Partition Table ${ }^{a}$

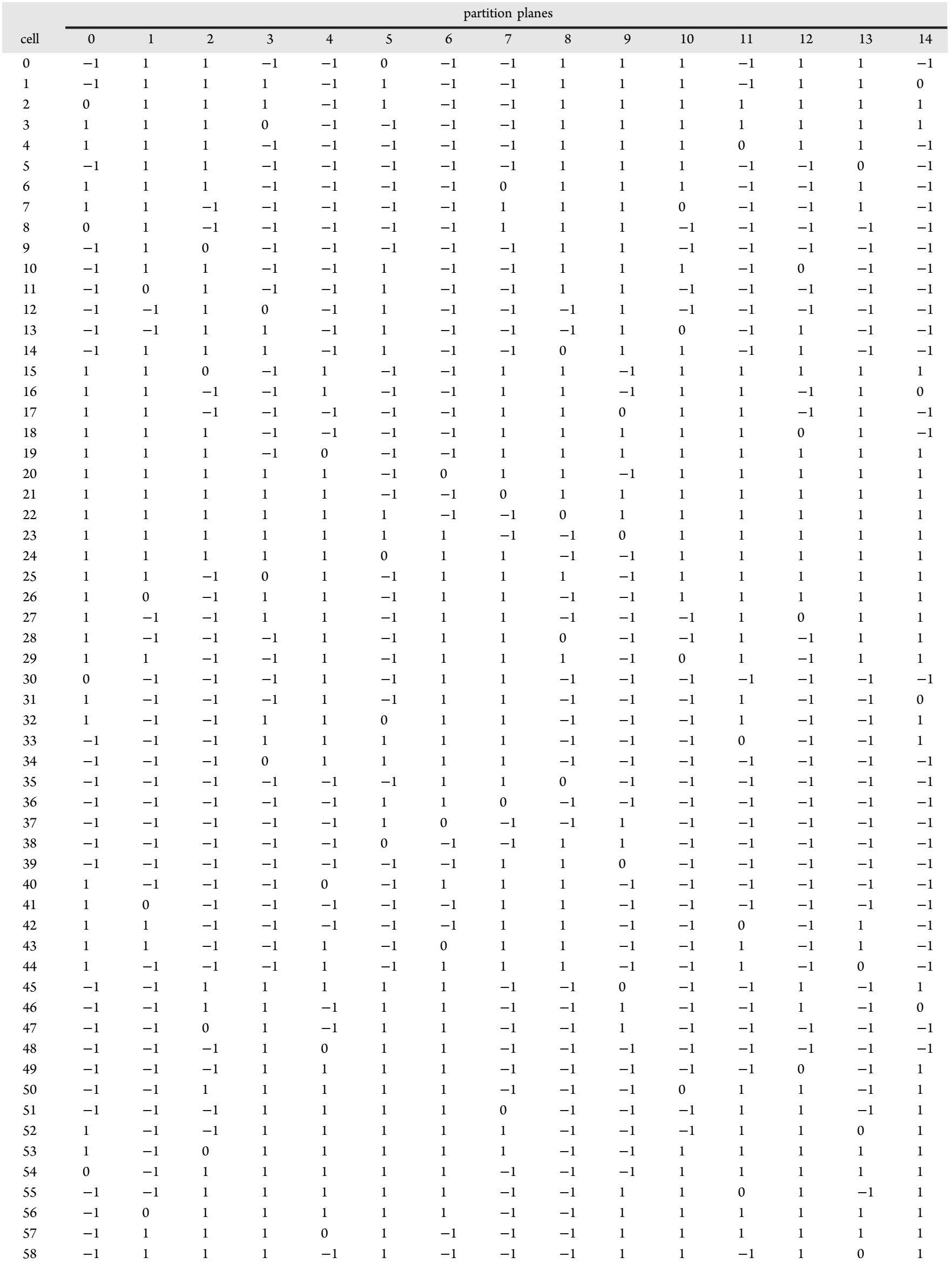

4460

DOI: $10.1021 /$ acsomega.8b00277
ACS Omega 2018, 3, 4458-4465 
Table 3. continued

\begin{tabular}{|c|c|c|c|c|c|c|c|c|c|c|c|c|c|c|c|}
\hline \multirow[b]{2}{*}{ cell } & \multicolumn{15}{|c|}{ partition planes } \\
\hline & 0 & 1 & 2 & 3 & 4 & 5 & 6 & 7 & 8 & 9 & 10 & 11 & 12 & 13 & 14 \\
\hline 59 & -1 & -1 & 1 & 1 & -1 & 1 & 0 & -1 & -1 & 1 & 1 & -1 & 1 & -1 & 1 \\
\hline
\end{tabular}

${ }^{a}$ The numbers 1 and -1 denote the upward and downward directions of the planes, respectively. The number 0 denotes both directions. The direction of the normal vectors in Table 1 is defined to indicate the upward direction.

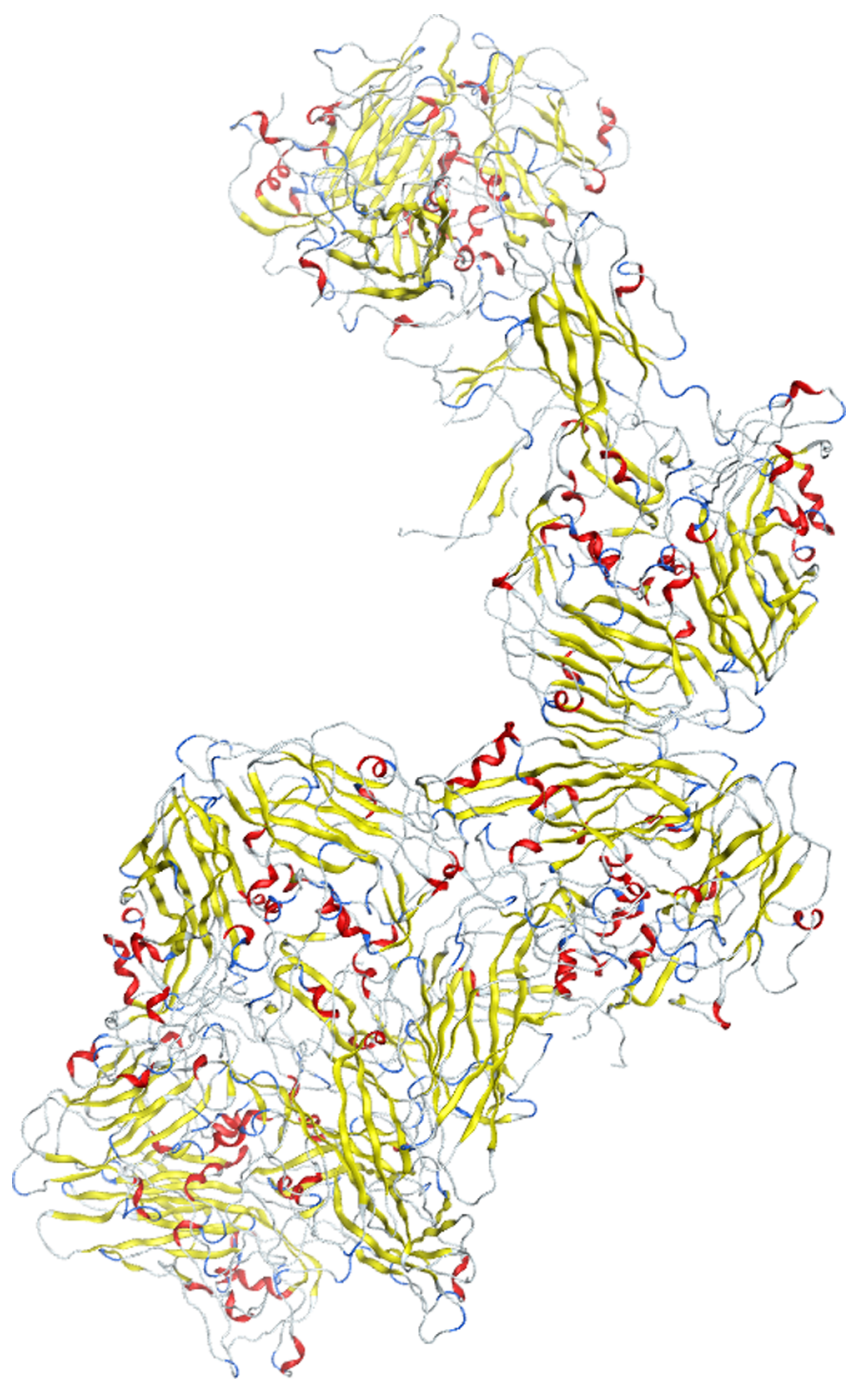

Figure 3. Structures of the protein units $0,2,5,6$, and 7 of the $2 \mathrm{PLV}$ of the poliovirus capsid generated by "capgen -c 0, 2, 5-7 -tp pdb2plv.ent", where heteroatoms are not shown. The direction of visualization is the same as that in Figure 1a.

subgroup of icosahedral group, $I$ ". The group $I$ is also written as "alternating group, $A_{5}$ ”. The multiplication of two rotation matrices, $R[q]$ and $R[r]$, is equal to another rotation matrix, as follows

$$
R[s]=R[q] R[r]
$$

Equation 3 can be expressed in an algebraic form as follows

$$
s \equiv q \otimes r
$$

where $\equiv$ and $\otimes$ denote equality and multiplication, respectively. The multiplication can be performed using the "group table". ${ }^{12,19}$ The neutral element is written as 0 in the present study. The inverse matrix can be written as follows, using a negative number for the rotation matrix index

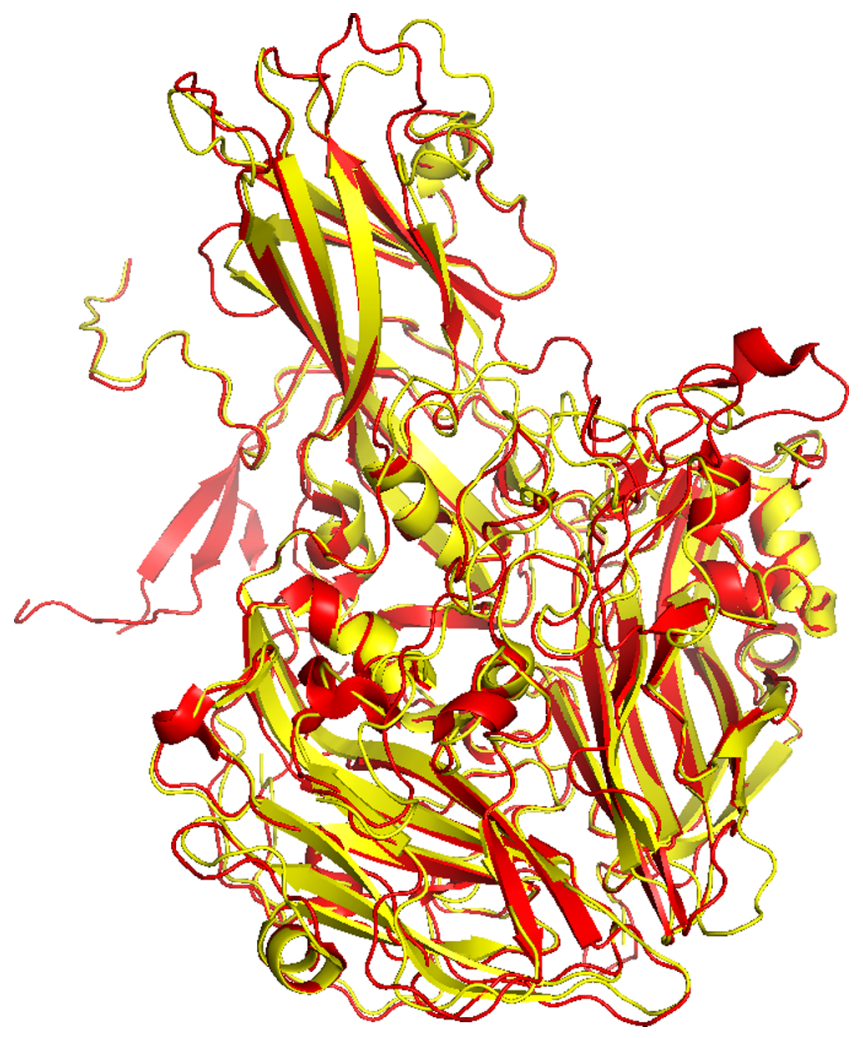

Figure 4. 4RHV structure was rotated using CAPROT onto the $2 \mathrm{PLV}$ position (red). The rotated 4RHV structure is shown in yellow. The direction of visualization is the same as that in Figure 1a.

$$
R^{-1}[d]=R[-d]
$$

Because the cell numbers and the rotation matrices are related by a one-to-one correspondence, the set of all cell numbers makes the group that is isomorphous to the group of rotation matrices, i.e., the group I. Similarly, the set of all protein unit numbers forms the group $I$. Thus, eq 5 is not only applicable to the rotation matrices but also to both the cell numbers and protein unit numbers. As shown in Figure 1, each cell is partitioned from the surrounding by four partition planes. It should be noted that the normal vector of each partition plane is equal to the rotation axis of one of the 2-fold rotation matrices. As there are 152 -fold rotation matrices, there are 15 partition planes. Each partition plane passes through the center of the capsid to bisect the entire capsid into upper and lower regions. Thus, cells are defined by the partition planes, as shown in Table 1 (partition table) of a previous article. ${ }^{12}$ The cell number for atom $k_{p}$ is written $c l\left(k_{p}\right)$, where $c l()$ is referred to as the "cell number function". Similarly, the "protomer number function", $\operatorname{pr}\left(k^{c}\right)$, returns the protein unit number of atom $k^{c}$. If the coordinates of atom $k_{p}$ are known, the coordinates of atom $k^{0}$ are determined according to the following "packing operation" 


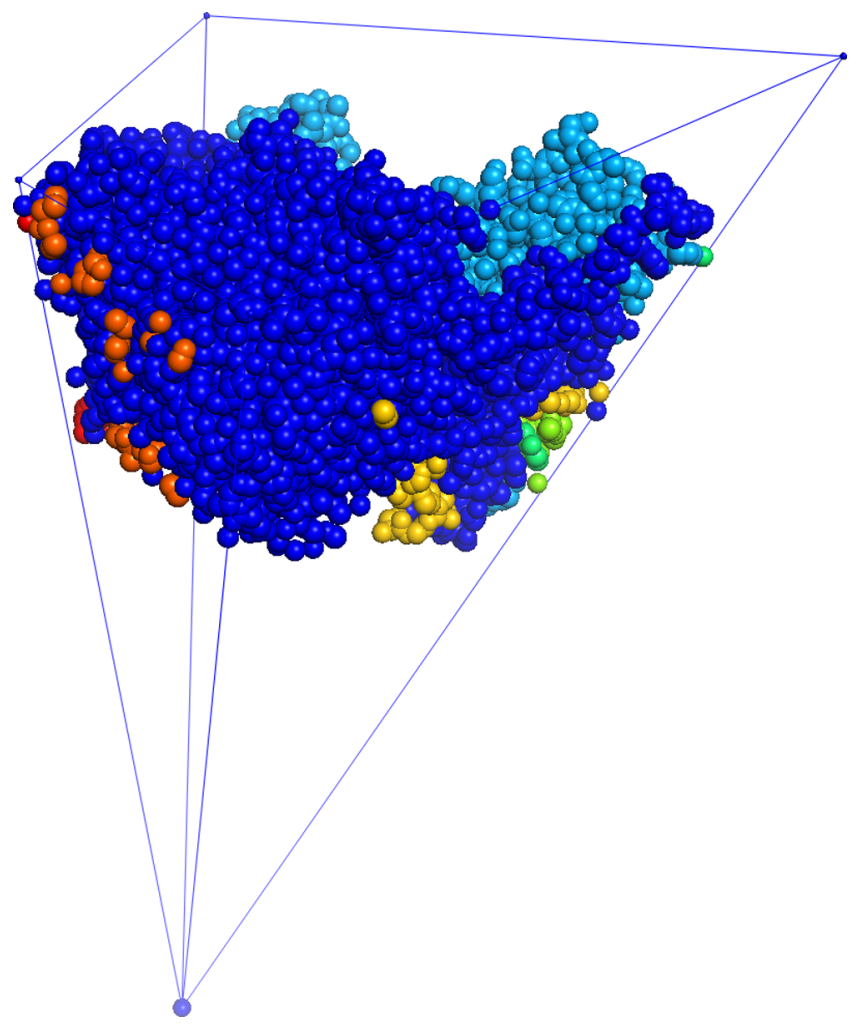

Figure 5. Graphics of the output of the packing operation by "cappack -v -tp pdb2plv.ent", where heteroatoms were deleted. The blue thin lines depict cell 0 . Atom colors correspond to the cell numbers.

$$
\mathbf{x}\left(k^{0}\right)=R\left[-c l\left(k_{p}\right)\right] \mathbf{x}\left(k_{p}\right)
$$

Applying the rotation matrix $R[c]$ to both sides, the coordinates of $k^{c}$ are determined as follows

$$
\mathbf{x}\left(k^{c}\right)=R[c] R\left[-c l\left(k_{p}\right)\right] \mathbf{x}\left(k_{p}\right)
$$

Thus

$$
\operatorname{pr}\left(k^{c}\right) \equiv c \otimes\left(-c l\left(k_{p}\right)\right) \otimes p
$$

If $p$ is zero

$$
\operatorname{pr}\left(k^{c}\right) \equiv c \otimes\left(-c l\left(k_{0}\right)\right)
$$

The protein unit number can be determined by eq 8 or 9 from the cell numbers, $c l\left(k_{p}\right)$ or $c l\left(k_{0}\right)$. Furthermore, to calculate intercell interactions, the "relative protomer number" for an atom pair $\left(i^{c}, j^{d}\right)$ is defined as $\operatorname{pr}\left(j^{d}\right) \otimes\left(-p r\left(i^{c}\right)\right)$. If $i^{c}$ and $j^{d}$ are connected with a bond, $c$ and $d$ should be equal; i.e., $\operatorname{pr}\left(j^{d}\right)$ $\otimes\left(-p r\left(i^{c}\right)\right)$ should be 0 (interprotein unit bonds are neglected for simplicity). From eq 9, the following is obtained

$$
p r\left(j^{d}\right) \otimes\left(-p r\left(i^{c}\right)\right) \equiv d \otimes\left(-c l\left(j_{0}\right)\right) \otimes c l\left(i_{0}\right) \otimes(-c)
$$

Letting $c$ be 0

$$
p r\left(j^{d}\right) \otimes\left(-p r\left(i^{0}\right)\right) \equiv d \otimes\left(-c l\left(j_{0}\right)\right) \otimes c l\left(i_{0}\right)
$$

If the left-hand side of the above is equal to 0

$$
d \equiv\left(-c l\left(i_{0}\right)\right) \otimes c l\left(j_{0}\right)
$$

Equation 12 presents a necessary and sufficient condition for atom pair $\left(i^{0}, j^{d}\right)$ to be an intraprotein unit pair. Equations
$8-12$, known as "protomer number equations", are necessary for the accelerated RSBC calculations using the packing operation.

The above mathematical devices have been implemented in the program APRICOT. ${ }^{12}$ However, our previous studies have been restricted to nine cells, including cell 0 . The complete mathematical and programing devices concerning all 60 cells are determined and implemented to CAPLIB, as shown in the following sections.

\section{THEORETICAL BASIS}

The coordinate system adopted for APRICOT and CAPLIB is shown in Figure 1a. This is equal to the " $\mathrm{Z}(2)-3-5-\mathrm{X}(2)$ " system of VIPER $\mathrm{db}^{20,21}$ and is used for many Protein Data Bank (PDB) files, ${ }^{22,23}$ including the 2 PLV file of poliovirus. It should be noted that the method for labeling the 60 cells is arbitrary, but the authors adopted a black-filled quadrangle (Figure 1a) for "cell 0", as in the 2PLV file. The other 59 cells were labeled using eq 1 , where the rotation matrices $\mathrm{R}[\mathrm{m}]$ were defined as follows

$$
R[m]=\mathrm{A}^{J} \mathrm{~B}^{K} \mathrm{C}^{L} \mathrm{D}^{M}
$$

where $\mathrm{A}, \mathrm{B}, \mathrm{C}$, and $\mathrm{D}$ are, respectively, the 2 -fold rotation matrix, the 2-fold rotation matrix, the 3-fold rotation matrix, and the 5-fold rotation matrix, as shown in Table 1 . The integers, $J, K, L$, and $M$ range from 0 to 1,0 to 1,0 to 2 , and 0 to 4 , respectively, and $m$ is equal to $M+5 L+15 K+30 J$. This labeling method for the rotation matrices (and thus the cells and rotation matrices) using eq 13 is not adopted in the present 2PLV file. Table S1 shows the group table for the rotation matrices. Figure 2 shows the development of the icosahedron surface depicting the cell labels. All partition planes are defined in Table 2 and are depicted in Figures S1 and S2. Table 3 shows the complete partition table.

\section{RESULTS AND DISCUSSION}

As described in the preceding section, we developed the software library CAPLIB. Most of the CAPLIB programs were written in $\mathrm{C}$ language for use on Linux and MacOS machines. The main modules of CAPLIB are CAPGEN, CAPROT, CAPAXIS, and CAPPACK. The interface scripts to the graphics software PyMOL ${ }^{24,25}$ were also developed in Python language.

CAPGEN reads a PDB file and outputs the file after the B factors of atoms are replaced with the cell numbers. CAPGEN runs by the Linux or MacOS command "capgen -c0 -v -tp file1 $>$ file2", where "capgen" is the name of the executable file of CAPGEN, "-c0" indicates that no rotations are applied, "-v" denotes that cell numbers should be printed, "-tp" denotes that the cell numbers are defined by eq 13 , ">” denotes the output redirection of Linux, and file 1 and file 2 are the input and output PDB files, respectively. CAPGEN can also generate the entire capsid structure by the command "capgen -c 0-59 -tp file1 > file2", where "-c 0-59" indicates that the entire capsid structure should be generated. Furthermore, CAPGEN can be used to generate any protein units selected by users. For example, the structure of the protein units $0,2,5,6$, and 7 can be generated by the command "capgen -c 0, 2, 5-7 -tp file1 > file2", as shown in Figure 3. The atomic coordinates in file 1 are rotated with $R[0], R[2], R[5], R[6]$, and $R[7]$ and stored in file2. When the option -tp is omitted, the labels of rotation matrices adopted in the BIOMT lines of "file1" are used. The option "-m 


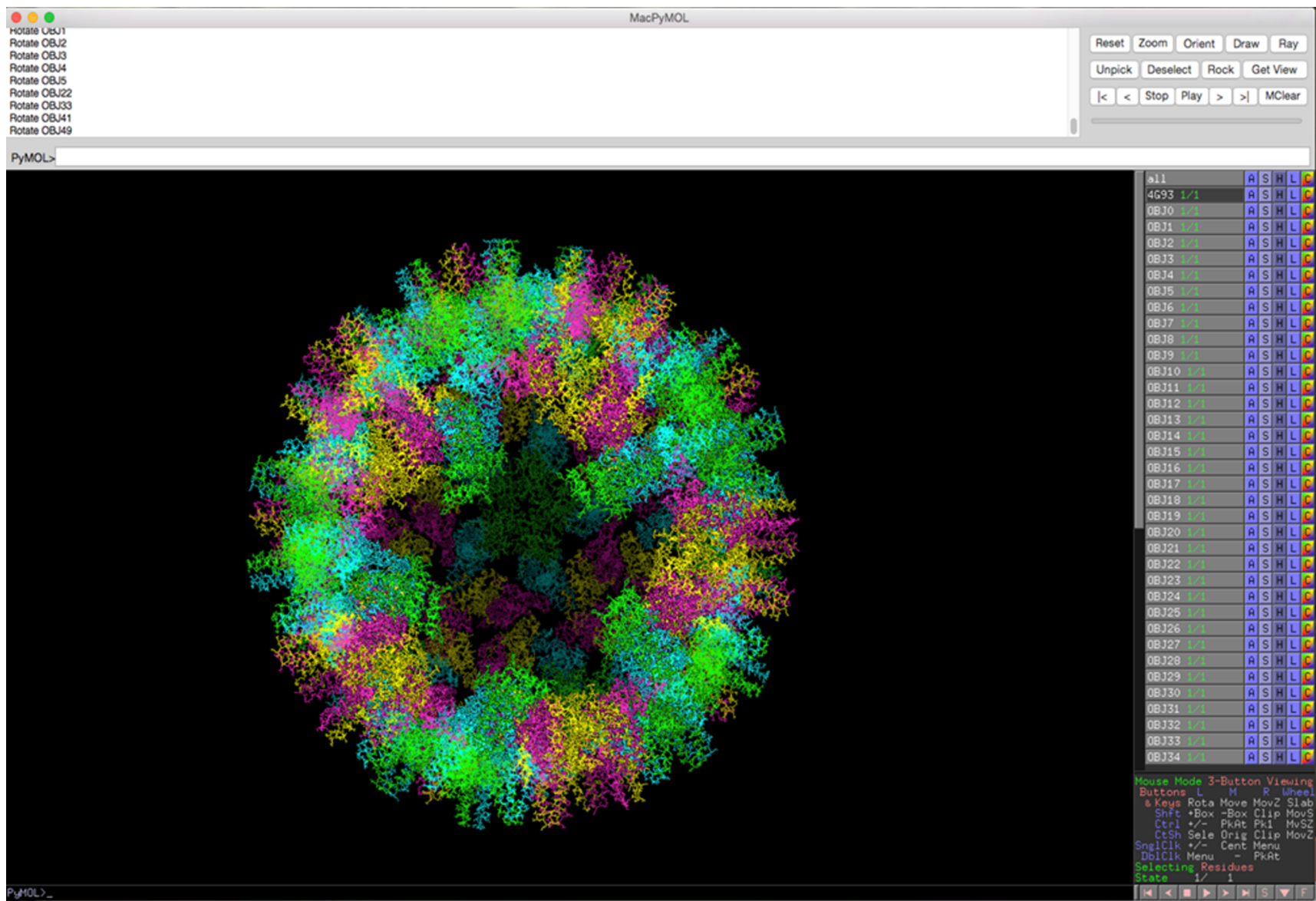

Figure 6. Entire structure of the human hepatitis B virus capsid, 4G93. Some protein units are hidden by clicking the PyMOL hidden buttons in the selection panel to show the inner structure of capsid.

fileR" indicates that the matrices written in "fileR" are used for the rotation by CAPGEN.

CAPAXIS reads a PDB file of icosahedrally symmetric capsids, which contain 60 rotation matrices in the "BIOMT" lines, and prints the rotation axes and angles adopted. Some PDB files of icosahedrally symmetric capsids have more than 60 matrices, but those files are not supported by CAPAXIS. If many PDB files are given to CAPAXIS, as "capaxis filela file1b file1c ...", the files are classified using the rotation axes. A sample of the results by CAPAXIS is shown in Figure S3. If option "-1" is added (i.e., "capaxis -1 file1 > file2"), CAPAXIS treats file 1 as a list of PDB file names. A sample of the classification of 458 PDB files is shown in Figure S4, where the PDB files were classified into 120 groups. The largest group was composed of the $229 \mathrm{PDB}$ files, including the 4RHV file of rhinovirus, and the second largest group was composed of 123 PDB files, including the 2PLV file of poliovirus. The third and fourth groups were composed of 47 and 21 PDB files, respectively. Other groups were composed of fewer than seven PDB files.

CAPROT reads two PDB files and analyzes their rotation axes. Thus, CAPAXIS moves the atoms in the first PDB file onto those of the second PDB file. For example, Figure 4 shows the results of "caprot pdb4rhv.ent pdb2plv.ent > output.pdb", in which the 4RHV structure was moved onto the $2 \mathrm{PLV}$ structure and the coordinate axes of the two proteins are identical. The translation vectors written in the PDB files are also considered. If the second PDB file is omitted and option -tp is added, the atoms are rotated onto the standard axes of the present study (Figure 1a). CAPROT is necessary for the comparison of capsid structures and for setting and analyzing RSBC MD simulations.

CAPPACK reads a PDB file and performs the packing operation of eq 6. If one enters the command "cappack file1 > file2", the atomic coordinates after the packing operation are stored in file2. If option -v is added, the B factors are replaced by the cell numbers, as shown in Figure 5. Although the packing operation is performed by APRICOT during MD simulations in RSBC, the packing operation only considers the atoms in the neighboring eight cells around cell 0 . In contrast, CAPPACK can perform the packing operation for any atom in any of the 60 cells.

The Python interface scripts were also developed to use the CAPLIB programs in the graphics software, PyMOL. For example, Figure 6 shows the entire structure of a capsid generated by the scripts. In Figure 6, a PDB file was first loaded to PyMOL and second, the script file was loaded by writing "run caplib.py" in the command window of PyMOL. Finally, by writing the name of the script, "capgen(1)", the CAPGEN module was called to generate the entire structure. The manipulation on each protein unit is simple because the protein units can be selected by clicking the object buttons in the selection panel at the right. The object buttons named "OBJ0", "OBJ1", "OBJ2", ... correspond to the protein units $0,1,2, \ldots$, respectively. Figure 7 shows the structure made by a manipulation that was composed of rotations. First, the 


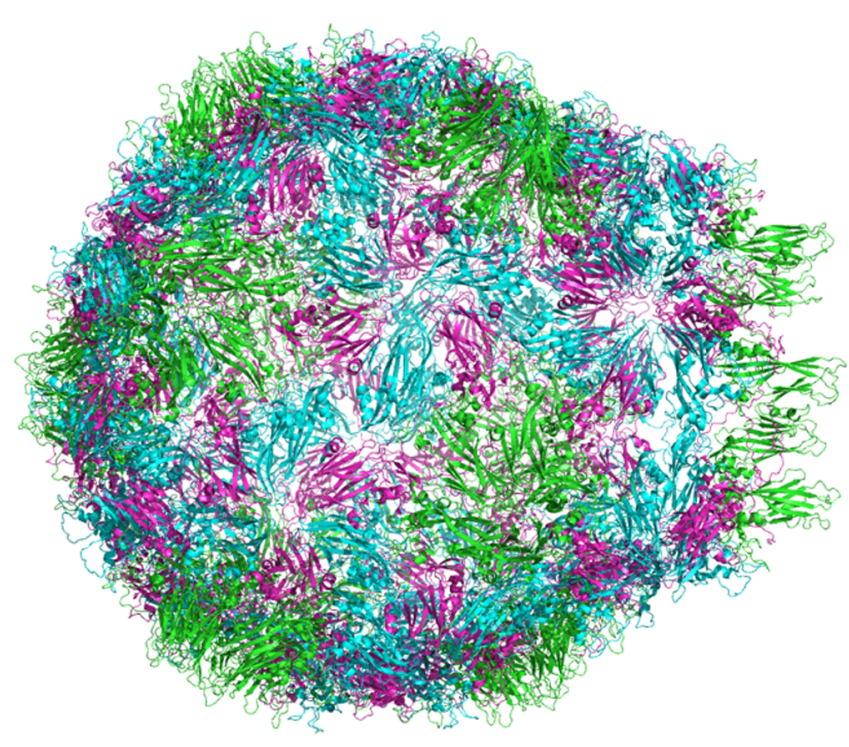

Figure 7. Set of rotations were applied to the human hepatitis B virus structure, 4G93, and a hole was made at the 5-fold axis.

standard axis for the manipulation was determined by writing the script name of "setaxis(OBJ2, OBJ1)" in the command window of PyMOL. Because OBJ2 and OBJ1 (i.e., protein units 2 and 1) are related with each other by the rotation at a 5-fold axis (see Figure 1a), this script selects the 5-fold axis for the standard axis. Second, by writing "capswch $(50,30)$ ", the vector to the center of each protein unit from the origin was calculated. If the angle between the vector and the standard axis is smaller than $50^{\circ}$, the protein unit was rotated by $30^{\circ}$ around its rotation axis that penetrates the center of the protein unit and is orthogonal to the standard axis. Therefore, only the protein units near the standard axis were rotated to make a hole at the standard axis.

This study presented the CAPLIB library equipped with the mathematical tables needed for the modeling and analysis on the complete 60 cells and protein units. By the $C$ programs and Python scripts of CAPLIB, it is possible to perform important calculations of modeling and analysis on the icosahedrally symmetric viral capsids. The source codes of the version $\beta$ of CAPLIB will be distributed under the 2 -clause BSD license ${ }^{26}$ after the publication of this article. ${ }^{27}$ The authors believe that the CAPLIB library can be widely used under this relatively loose license for the studies on the icosahedrally symmetric capsids.

\section{ASSOCIATED CONTENT}

\section{S Supporting Information}

The Supporting Information is available free of charge on the ACS Publications website at DOI: 10.1021/acsomega.8b00277.

Group table; partition planes $0-7$ on the icosahedron surface; partition planes 8-14 on the icosahedron surface; rotation axes and angles of the $4 \mathrm{RHV}$ file; 458 PDB files classified by CAPAXIS (PDF)

\section{AUTHOR INFORMATION}

\section{Corresponding Author}

*E-mail: syoneda@kitasato-u.ac.jp.

ORCID $\odot$

Shigetaka Yoneda: 0000-0001-5554-2710
Go Watanabe: 0000-0001-6713-1249

\section{Notes}

The authors declare no competing financial interest.

\section{ACKNOWLEDGMENTS}

The authors appreciate the use of computer resources in the Research Center for Computational Science, Okazaki, Japan.

\section{REFERENCES}

(1) Crick, F. H. C.; Watson, J. D. Structure of Small Viruses. Nature 1956, 177, 473-475.

(2) Casper, D. L. D.; Klug, A. Physical Principles in the Construction of Regular Viruses. Cold Spring Harbor Symp. Quant. Biol. 1962, 27, 124.

(3) Twarock, R. Mathematical Virology: a Novel Approach to the Structure and Assembly of Viruses. Philos. Trans. R. Soc., A 2006, 364, $3357-3373$

(4) Zlotnick, A. To Build a Virus Capsid. An Equilibrium Model of the Self Assembly of Polyhedral Protein Complexes. J. Mol. Biol. 1994, $241,59-67$.

(5) Keef, T.; Micheletti, C.; Twarock, R. Master Equation Approach to the Assembly of Viral Capsids. J. Theor. Biol. 2006, 242, 713-712.

(6) Kerner, R. Self-Assembly of Icosahedral Viral Capsids: the Combinatorial Analysis Approach. Math. Modell. Nat. Phenom. 2011, 6, 136-158.

(7) Dykeman, E. C.; Stockley, P. G.; Twarock, R. Building a Viral Capsid in the Presence of Genomic RNA. Phys. Rev. E 2013, 87, No. 022717.

(8) Bricogne, G. Methods and Programs for Direct-Space Exploitation of Geometric Redundancies. Acta Crystallogr., Sect. A: Cryst. Phys., Diffr., Theor. Gen. Crystallogr. 1976, 32, 832-847.

(9) Zhao, G.; Perilla, J. R.; Yufenyuy, E. L.; Meng, X.; Chen, B.; Ning, J.; Ahn, J.; Gronenborn, A. M.; Schulten, K.; Aiken, C.; Zhang, P. Mature HIV-1 Capsid Structure by Cryo-Electron Microscopy and AllAtom Molecular Dynamics. Nature 2013, 497, 643-646.

(10) Phelps, D. K.; Speelman, B.; Post, C. B. Theoretical studies of viral capsid proteins. Curr. Opin. Struct. Biol. 2000, 10, 170-173.

(11) Çağin, T.; Holder, M.; Pettitt, B. M. A Method for Modeling Icosahedral Virions; Rotational Symmetry Boundary Conditions. J. Comput. Chem. 1991, 12, 627-634.

(12) Yoneda, S.; Kitazawa, M.; Umeyama, H. Molecular Dynamics Simulation of a Rhinoviral Capsid under Rotational Symmetry Boundary Conditions. J. Comput. Chem. 1996, 17, 191-203.

(13) Yoneda, S.; Umeyama, H. Free Energy Perturbation Calculations on Multiple Mutation Bases. J. Chem. Phys. 1992, 97, 6730-6736.

(14) Yoneda, T.; Yoneda, S.; Takayama, N.; Kitazawa, M.; Umeyama, H. A Homology Modeling Method of an Icosahedral Viral Capsid: Inclusion of Surrounding Protein Structures. J. Mol. Graphics Modell. 1999, 17, 114-119.

(15) Azuma, H.; Yoneda, S. Structure and Dynamics of the GH Loop of the Foot-and-Mouth Disease Virus Capsid. J. Mol. Graphics Modell. 2009, 28, 278-286.

(16) Watanabe, G.; Sato, S.; Iwadate, M.; Umeyama, H.; Hayakawa, M.; Murakami, Y.; Yoneda, S. Molecular Dynamics Simulations to Determine the Structure and Dynamics of Hepatitis B Virus Capsid Bound to a Novel Anti-viral Drug. Chem. Pharm. Bull. 2016, 64, 13931396.

(17) Yoneda, S. A Further Implementation of the Rotational Symmetry Boundary Conditions for Calculations of P43212 Symmetry Crystals. J. Mol. Graphics Modell. 1997, 15, 233-237.

(18) Roy, A.; Post, C. B. Micoroscopic Symmetry Imposed by Rotational Symmetry Boundary Conditions in Molecular Dynamics Simulations. J. Chem. Theory Comput. 2011, 7, 3346-3353.

(19) Hardy, F. L. Elementary Exercises in Group Theory; Charles E. Merill Publishing Co.: Columbus, Ohio, 1970.

(20) Reddy, V. S.; Natarajan, P.; Okerberg, B.; Li, K.; Damodaran, K. V.; Morton, R. T.; Brooks, C. L., III; Johnson, J. E. Virus Particle 
Explorer (VIPER), a Website for Virus Capsid Structures and Their Computational Analysis. J. Virol. 2001, 75, 11943-11947.

(21) Carrillo-Tripp, M.; Shepherd, C. M.; Borelli, I. A.; Venkataraman, S.; Lander, G.; Natarajan, P.; Johnson, J. E.; Brooks, C. L., III; Reddy, V. S. VIPERdb2: an Enhanced and Web API Enabled Relational Database for Structural Virology. Nucleic Acids Res. 2009, 37, D436-D442.

(22) Sussman, J. L.; Lin, D.; Jiang, J.; Manning, N. O.; Prilusky, J.; Ritter, O.; Abola, E. E. Protein Data Bank (PDB): Database of ThreeDimensional Structural Information of Biological Macromolecules. Acta Crystallogr., Sect. D: Biol. Crystallogr. 1998, 54, 1078-1084.

(23) Worldwide Protein Data Bank Home Page. http://www.wwpdb. org/documentation/file-format (accessed March 17, 2018).

(24) The PyMOL Molecular Graphics System, version 2.0; Schrödinger, LLC. https://pymol.org/2/ (accessed March 17, 2018).

(25) The PyMOLWiki Page. https://pymolwiki.org/index.php/ Main Page (accessed March, 2018).

(26) Open Source Initiative Home Page. https://opensource.org/ licenses/BSD-2-Clause (accessed March 17, 2018).

(27) GitHub Page of CAPLIB. https://github.com/picorna/caplib (2018). 